\title{
Dual isotope simultaneous imaging to evaluate the effects of intracoronary bone marrow-derived mesenchymal stem cells on perfusion and metabolism in canines with acute myocardial infarction
}

\author{
LINJUN HAO ${ }^{1}$, JIN HAO $^{2}$, WEI FANG ${ }^{3}$, CHUNLEI HAN $^{4}$, KAIXIU ZHANG $^{5}$ and XUEMEI WANG ${ }^{5}$ \\ ${ }^{1}$ Department of Health Care Center, Affiliated Hospital of Inner Mongolia Medical University; ${ }^{2}$ Medical Laboratory Class \\ of 2013 Grade, Inner Mongolia Medical University, Hohhot 010050; ${ }^{3}$ Department of Nuclear Medicine, \\ Cardiovascular Institute and Fu Wai Hospital, Beijing Union Medical University, Beijing 100037, P.R. China; \\ ${ }^{4}$ Turku PET Centre, Turku University Hospital, Turku FI-20521, Finland; ${ }^{5}$ Department of Nuclear Medicine, \\ Affiliated Hospital of Inner Mongolia Medical University, Hohhot 010050, P.R. China
}

Received March 24, 2015; Accepted May 5, 2015

DOI: $10.3892 /$ br.2015.474

\begin{abstract}
Stem cell therapy on acute myocardial infarction (AMI) has been performed for over a decade. In the present study, cardiac perfusion, metabolism and function in dogs with AMI treated by intracoronary injection of bone marrow-derived mesenchymal stem cells (MSCs) were evaluated by dual isotope simultaneous acquisition (DISA) of single positron emission computed tomography (SPECT). Dogs $(n=12,20-30 \mathrm{~kg})$ were randomly assigned to two groups: A graft study $(n=6)$ and control group $(n=6)$. Bone marrow mesenchymal aspirate was collected 3 weeks before surgical procedure. Stem cells were induced by 5 -azacytidine for differentiation into myocytes. The dog AMI model was produced by blocking the blood stream at $1 / 3$ of the distinct left anterior descending coronary artery for $90 \mathrm{~min}$. For dogs in the grafting group, MSCs were transplanted by intracoronary injection, and for the control group, $0.9 \% \mathrm{NaCl}$ was injected instead. At 1 and 10 weeks after MSCs were grafted, respectively, SPECT DISA was performed for each dog in the two groups with ${ }^{99 \mathrm{~m} T c-S P E C T ~ M I B I ~}(925 \mathrm{MBq})$ and ${ }^{18}$ F-FDG $(222 \mathrm{MBq}$ ) for evaluation of myocardial perfusion and metabolism. After the dogs were sacrificed, heart tissue was stained by myocyte-specific antibodies for newborn vessels, troponin $\mathrm{T}$ and bromodeoxyuridine (BrdU). Following induction by 5 -azacytidine, the morphological features with
\end{abstract}

Correspondence to: Professor Xuemei Wang, Department of Nuclear Medicine, Affiliated Hospital of Inner Mongolia Medical University, 1st North Tongdao Street, Hohhot 010050, P.R. China E-mail: wangxuemei201010@163.com

Key words: perfusion, metabolism, stem cell, acute myocardial infarction colony formation, microfilament, as well as atrial granules and positive stainings of $\alpha$-actinin, myosin and troponin I demonstrated strongly that the MSCs differentiated into myocytes. The number of viable myocardial segments was 10 in the grafting group, which was significantly greater compared with the control group. The ejection fraction of the infarcted left ventricle $(\mathrm{LVEF}, \%)$ increased from $53.80 \pm 9.58$ to $70.00 \pm 7.52$ (change, 16.20 \pm 2.93 ) at 1 and 10 weeks after MSCs engraftment, whilst in the control group, LVEF was 50.50 \pm 8.02

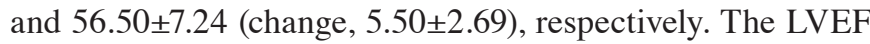
difference was statistically significant $(\mathrm{P}<0.05)$ between the graft and control groups. Furthermore, immunostaining of all the myocyte-specific antibodies (for newly born vessels, troponin $\mathrm{T}$ and BrdU) was positive. In conclusion, direct intracoronary injection of bone marrow MSCs into injured myocardium in the experimental dog AMI model can significantly improve cardiac function with new vessel formation and myocyte-specific biomarker expression, and in particular, the present study further shows that DISA SPECT can be used for the assessment of stem cell transplantation in the heart.

\section{Introduction}

Acute myocardial infarction (AMI) remains the leading cause of morbidity, mortality and heart failure worldwide. Over the past few decades, significant efforts have been made in the treatment of AMI based on early reperfusion of the culprit artery, including pharmacological and mechanical therapies, resulting in significantly reducing mortality (1). However, the lack of repair of a substantial amount of damaged cardiac tissue may lead to congestive heart failure. Necrosis or scarred tissue following AMI in the heart will lead to significant ventricular remodeling characterized by dilation of the left ventricular cavity, thinning of the infarcted tissue and electrical remodeling, resulting in the increase of the risk of sudden cardiac fatality. The use of stem cells with angiogenic properties for therapy of AMI has been widely investigated as a feasible 
strategy for repairing injured myocardial muscle tissue $(2,3)$. Mesenchymal stem cells (MSCs), as a subpopulation of bone marrow cells, were found to differentiate into various cell types, including functional cardiomyocytes. MSCs are easily isolated, have a proclivity for ex vivo expansion and a potential for allogeneic use (4). MSC therapy for AMI has been investigated widely in animals and humans $(5,6)$.

Positron emission tomography (PET) has been applied to investigate the improvement of myocardial function following stem cell therapy for AMI in humans and animals as a non-invasive imaging tool, such as imaging of ${ }^{11} \mathrm{C}$-acetate, ${ }^{13} \mathrm{~N}$-ammonia and ${ }^{18} \mathrm{~F}$-FDG (7). These PET tracers are not always available for numerous hospitals as their generation requires the on-site cyclotron (except ${ }^{18}$ F-FDG) $(8,9)$. Compared with PET, single proton emission computed tomography (SPECT) is much more available in practices. In particular, SPECT with dual isotope simultaneous acquisition (DISA) allowed the assessment of myocardial perfusion (with ${ }^{99 \mathrm{~m}} \mathrm{Tc}-\mathrm{MIBI}$ ) and metabolism (with $\left.{ }^{18} \mathrm{~F}-\mathrm{FDG}\right)$ in a single study by Slart et al $(10,11)$. Therefore, DISA SPECT provides an easy procedure for stem cell study to assess myocardial function for humans and animals. Thus, the present study was designed to evaluate the engraftment in myocardium and the improvement of cardiac function following intracoronary injection of pre-differentiated MSCs in a canine model with induced AMI using DISA SPECT, to quantify myocardial perfusion and metabolism, respectively.

\section{Materials and methods}

Animal model of myocardial infarction. All the surgical procedures and postoperative care were performed in accordance with the guidelines of the Beijing Fuwai Hospital Animal Care and Use Committee (Beijing, China). All the study protocols were approved by the Ethics Committee of Beijing Fuwai Hospital. A total of 12 dogs (male and female, 2-3 years old and 20-30 kg) were included in the experiment: 6 were the control group and another 6 were the graft group. All the dogs in the two groups were anesthetized by venous injection of ketamine $(50 \mathrm{mg} / \mathrm{kg})$ and diazepam $(0.05 \mathrm{mg} / \mathrm{kg})$. Following the induction of anesthesia, the animals were intubated and mechanically ventilated through a small-animal ventilator with a minute volume of 1.5-2.5 ml. Respiratory rate and tidal volume were adjusted according to body weight and other physiological parameters. To avoid heat loss, the body temperature was maintained by a heating lamp and the animals were covered with drapes, leaving only the necessary surgical area uncovered. The electrocardiogram was monitored throughout the surgery. Subsequent to the opening of the thoracic cavity and pericardium, four silk suture strings were placed underneath the vessel and the surrounding myocardium at the distal left anterior descending (LAD) coronary artery. LAD was ligated for $5 \mathrm{~min}$ and the perfusion was restored for $10 \mathrm{~min}$. After this, ligation was maintained for $90 \mathrm{~min}$, and LAD perfusion was restored again. Duration ligation, a small piece of a polyethylene tube was used to avoid damage to the vessel.

Bone marrow harvest, stem cells isolation, expansion and labeling. Four weeks before surgery, 10-15 ml bone marrow aspirate was collected in a syringe with $1 \mathrm{ml}$ heparin from the iliac crest from each dog after anesthesia was administrated.
Bone marrow mononuclear cells were isolated using Ficoll-Hypaque solution (1.077 g/ml; Sigma-Aldrich, St. Louis, MI, USA) and plated in Dulbecco's modified Eagle's medium (DMEM) containing 10\% fetal bovine serum (FBS) and antibiotics (100 U/ml penicillin and $100 \mu \mathrm{g} / \mathrm{ml}$ streptomycin; Invitrogen, Carlsbad, CA, USA). The media were changed and maintained in DMEM-F12 medium supplemented with $10 \%$ FBS and antibiotics for several weeks. To induce cell differentiation, cells were incubated in DMEM-F12 medium supplemented with 5\% FBS and antibiotics $24 \mathrm{~h}$ before treatment with 5-azacytidine ( $20 \mu \mathrm{mol} / \mathrm{l}$; Sigma-Aldrich) from the third passage. Following incubation, media were changed and maintained in DMEM-F12 supplemented with 10\% FBS and antibiotics for several weeks.

Cell differentiation was evaluated morphologically and immunohistochemically. Briefly, after 4 weeks of culture, differentiated cells were evaluated by phase contrast microscopy and electron microscopy, respectively. Furthermore, cultured cells were immunostained by monoclonal antibodies to sarcomeric $\alpha$-actine, cardiac myosin heavy chain and troponin I (Sigma-Aldrich).

Cellular transplant. For the graft group, at 2 or $3 \mathrm{~h}$ after perfusion was restored following 90 min ligation of LAD, 2-3 ml ( $10^{7}$ cells) of in vitro culture-expanded MSCs were implanted to the AMI area by intracoronary injection using a thin syringe. To identify the grafted cells in the host myocardium, one-third of the implanted MSCs were labeled with bromodeoxyuridine (BrdU) prior to implantation.

For dogs in the control group, $0.9 \% \mathrm{NaCl}$ was injected in the same way. Electrocardiography (ECG) was performed during surgical procedure and continued to 24-48 h.

Myocardial perfusion and metabolism assessment by SPECT. DISA SPECT with ${ }^{18} \mathrm{~F}-\mathrm{FDG}$ and ${ }^{99 \mathrm{~m}} \mathrm{Tc}-\mathrm{MIBI}$ was employed to assess myocardial perfusion and metabolism simultaneously, using a double-head $\gamma$-camera equipped with ultra-high-energy collimators (VariCam; GE Healthcare, Cleveland, OH, USA). The imaging protocol was as previously described [Slart et al (11) and De Boer et al (12)]. Briefly, at week 10 after implantation, ${ }^{99 \mathrm{~m}} \mathrm{Tc}-\mathrm{MIBI}(925 \mathrm{MBq})$ and ${ }^{18} \mathrm{~F}-\mathrm{FDG}$ (222 MBq) were injected into the ear vein of a dog after administration of anesthesia, and scanning started at 60-90 min after injection. The acquisition was set to continuous mode with a $64 \times 64$ word matrix. Dual isotope acquisition was obtained with two separate energy windows: $140 \mathrm{keV}$ with $20 \%$ window and $511 \mathrm{keV}$ with $20 \%$ window. Following this, a standard gated SPECT myocardial perfusion scan was performed for LVEF measurement.

Image analysis. Myocardial perfusion and metabolic imaging data were analyzed semi-quantitatively by two experienced nuclear cardiologists independently, with a five-point scoring system: 0 , normal uptake; 1 , mild hypoperfusion; 2 , moderate hypoperfusion; 3 , severe hypoperfusion; and 4, no uptake. Two cardiologists were blinded to the experimental groups.

Histology, pathology and immunohistochemistry. All the dogs were sacrificed following SPECT imaging. The heart was arrested by infusion of cadmium chloride $(0.1 \mathrm{M})$ into 
A

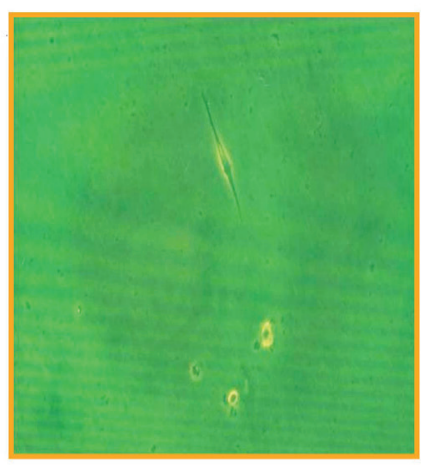

B

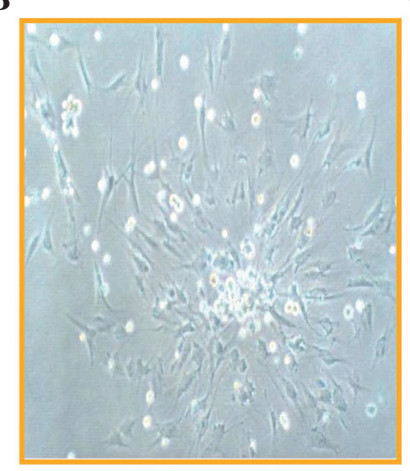

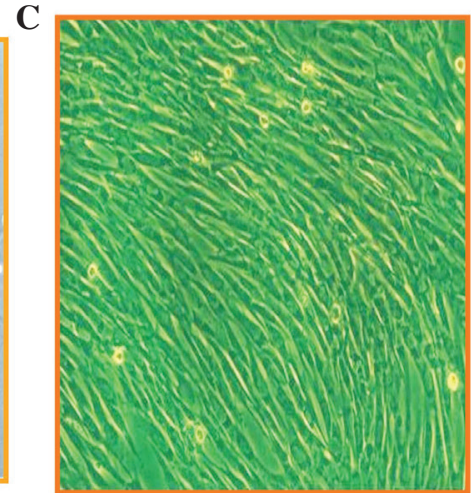

Figure 1. Photomicrographs of cultured mesenchymal stem cells (MSCs) with characteristics of MSCs in morphology and proliferation. (A) A single stem cell. (B) Colony formation of stem cells. (C) Spindle-like stem cell arrangement.

A

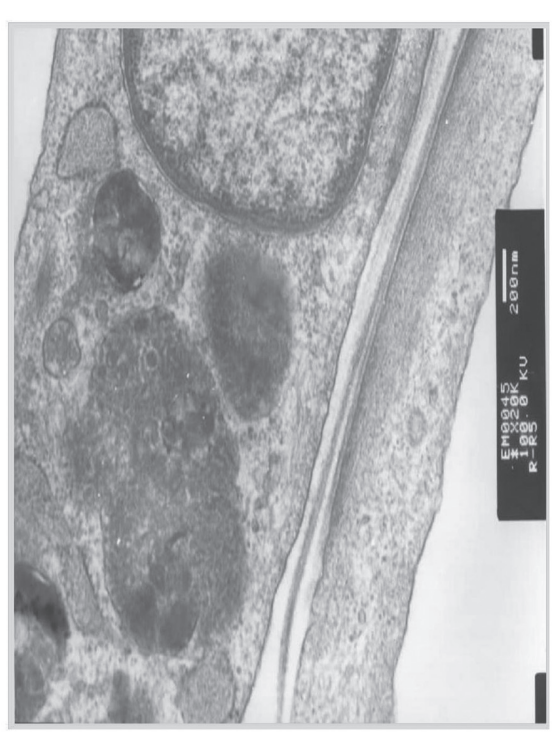

B

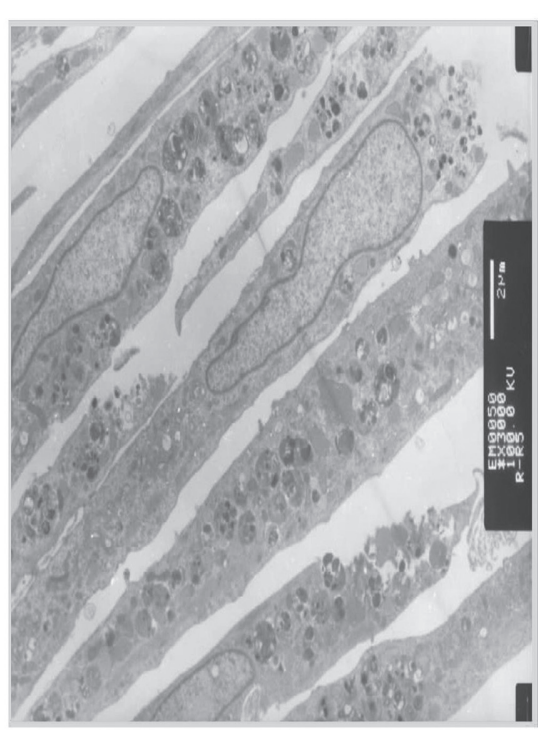

Figure 2. Electron micrographs of differentiated mesenchymal stem cells (MSCs) characterized with (A) microfilaments and (B) atrial granules around the nucleus.

the left atrium. The heart and circulation were perfused with phosphate-buffered saline [PBS (pH 7.4) $50 \mathrm{nM}$ sodium nitroprusside] and 5\% formalin in PBS at a physiological pressure for $10 \mathrm{~min}$. Subsequently, the hearts were dissected and immersion-fixed in 10\% formalin in PBS for $24 \mathrm{~h}$, processed and embedded in paraffin for further morphological, histological and immunohistochemical analyses. The heart was cut longitudinally, perpendicular to the infarcted area, resulting in an anterior and posterior section. From the two sections, $10 \mu \mathrm{m}$ slices were cut.

Statistical analysis. Differences among groups were compared using one-way analysis of variance with Student's t-test using SPSS v.12 software (SPSS, Inc., Chicago, IL, USA). P $<0.05$ was considered to indicate a statistically significant difference.

\section{Results}

Mesenchymal stem cell culture and differentiation. Differentiation of mesenchymal stem cells to myocytes induced by 5 -azacytidine was evaluated by microscopic and electron microscopic observations, as well as immunohistochemical staining. Fig. 1 shows the morphological changes following induction, from rod-like (Fig. 1A) to colony formation (Fig. 1B) and spindle-like cells (Fig. 1C). Using an electron microscope, microfilaments and atrial granules around the nucleus were identified (Fig. 2). Furthermore, by immunohistochemical staining, sarcomeric $\alpha$-actinin, cardiac myosin heavy head and troponin I were positive (Fig. 3). These observations confirmed that bone marrow mesenchymal stem cells have differentiated into cardiomyocytes.

Serum enzyme increase following ligation of LAD. The levels of serum enzymes after 9-12 h following LAD ligation, including creatine kinase (CK), isoenzymes of creatine kinase (CK-MB), glutamic oxaloacetic transaminase (GOT), glutamate-pyruvate transaminase (GPT) and troponine T (TNT), were significantly increased, as shown in Table I, in the two groups. High levels of cardiomyocytes enzyme in the serum indicated that myocardial ischemia or AMI occurred in the dogs of the two groups.

Myocardial function changes following MSCs implantation. One myocardial viability case is shown in Fig. 4 at different 

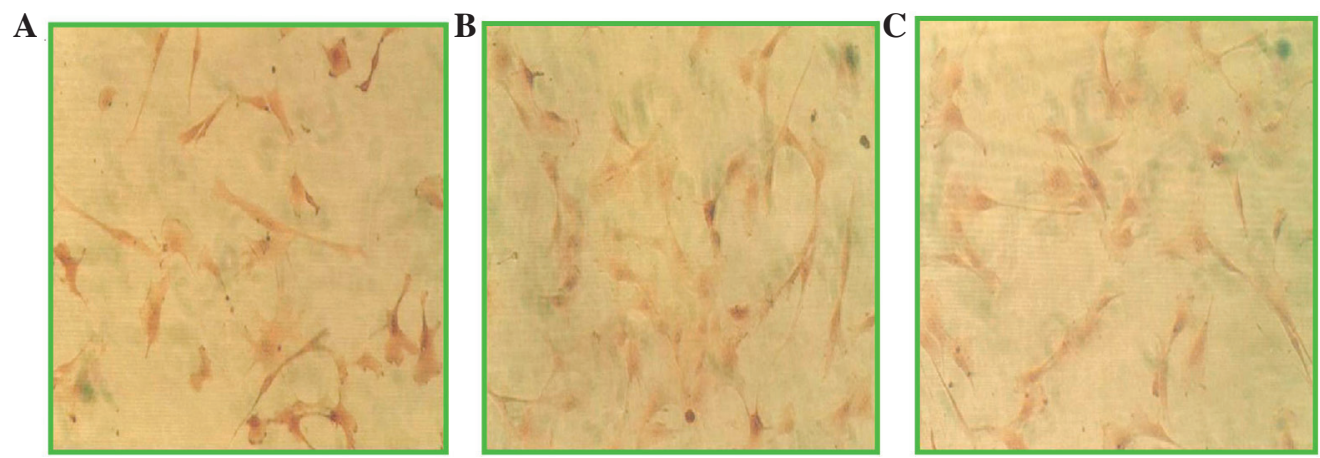

Figure 3. Photomicrographs of cultured mesenchymal stem cells (MSCs) immunohistochemically stained for cardiac sarcomeric (A) $\alpha$-actinin, (B) myosin heavy head and $(\mathrm{C})$ troponin I.

A
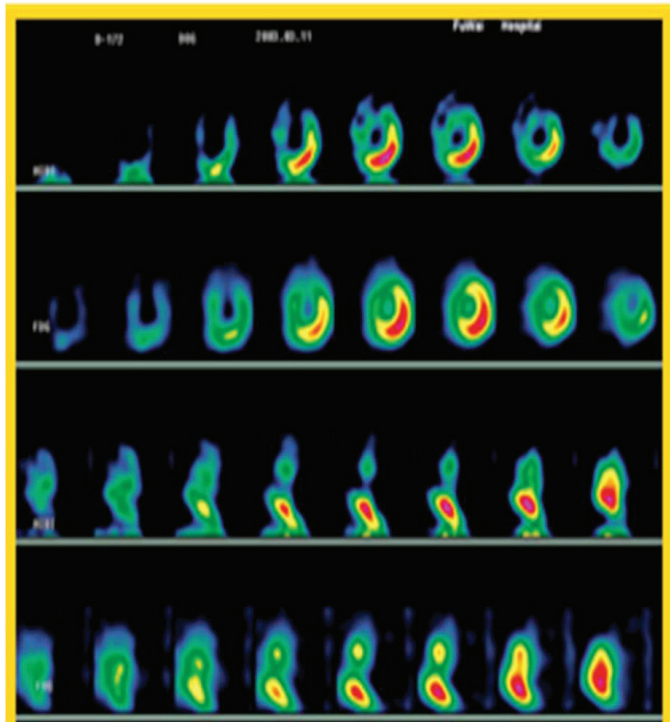

$00101010 \mathrm{Mn}$

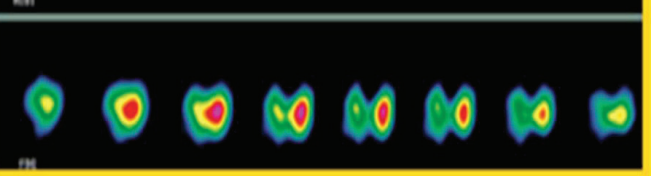

B
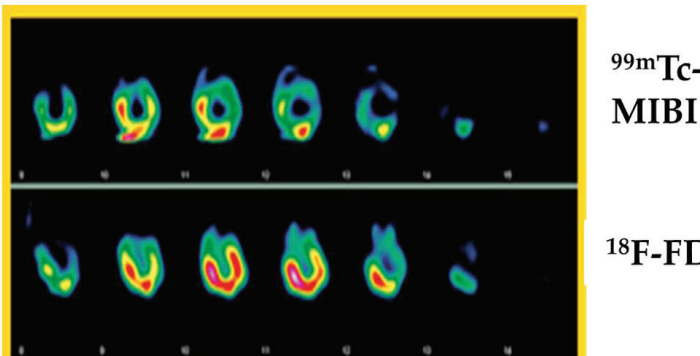

${ }^{18}$ F-FDG

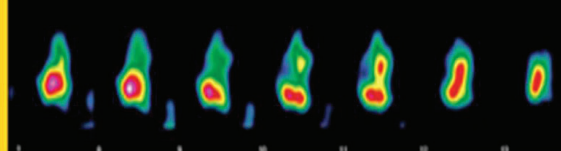

${ }^{99 m} \mathrm{Tc}-$

MIBI

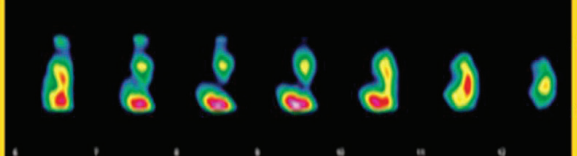

${ }^{18}$ F-FDG

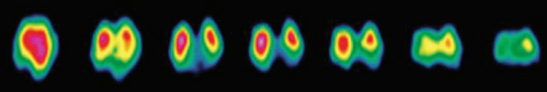

99mTc-

MIBI

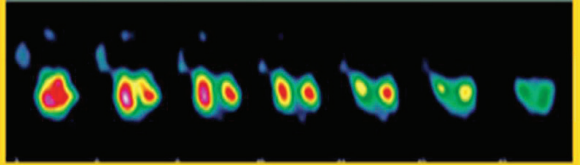

Figure 4. Myocardial perfusion and metabolic images from DISP single positron emission computed tomography (SPECT). Imaging was performed at (A) 1 and (B) 10 weeks after ligation of descending coronary artery (LAD). ${ }^{99 \mathrm{~m}} \mathrm{Tc}-\mathrm{MIBI}$ represents myocardial perfusion imaging and ${ }^{18} \mathrm{~F}-\mathrm{FDG}$ represents myocardial metabolic imaging.
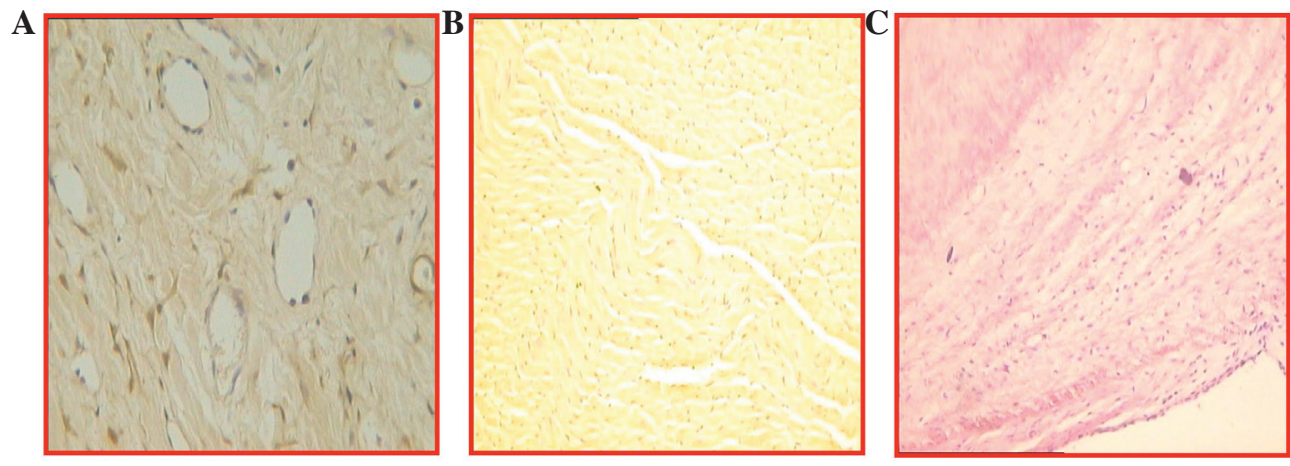

Figure 5. Photomicrographs of infarcted myocardium implanted with mesenchymal stem cells (MSCs) at 10 weeks after cell transplantation. Tissue sections were positively immunohistochemically stained for (A) BrdU (angiogenesis), (B) troponin I and (C) H\&E (lymphocytic infiltration). 
Table I. Levels of serum enzymes at 9-12 h after LAD ligation.

\begin{tabular}{lccccc}
\hline Group & CK, IU/l & CK-MB, IU/1 & GOT, IU/1 & GPT, IU/1 & TNT \\
\hline Control & $4,998.6 \pm 198.4$ & $423.7 \pm 67.9$ & $494.8 \pm 36.7$ & $231.3 \pm 45.6$ & +++ \\
Graft & $4,987.5 \pm 158.3$ & $428.3 \pm 58.2$ & $487.6 \pm 40.4$ & $235.4 \pm 41.8$ & +++ \\
\hline
\end{tabular}

No statistically significant difference between the control and graft groups. LAD, left anterior descending; CK, creatine kinase; CK-MB, isoenzymes of creatine kinase; GOT, glutamic oxaloacetic transaminase; GPT, glutamate-pyruvate transaminase; TNT, troponin T.

time points [1 (Fig. 4A) and 4 weeks (Fig. 4B)] after ligation of LAD. The change in the number of viable/infarcted segments in myocardium is presented as a summary in Table II. The infarcted number of myocardial segments was decreased significantly in the graft group, from 25 (1 week) to 15 (10 weeks), following MSCs implantation, indicating that myocardial regeneration occurred in the infarction area. Cardiac function was also improved significantly in the graft group, as LVEF increased from $53.80 \%$ (1 week) to $70.00 \%$ (10 weeks), but in the control group, LVEF had a small increase (from 50.5 to $56.5 \%$ ) at the same time-points, as shown in Table III.

Immunohistochemical staining. Evidence from immunohistochemical staining demonstrated the engraftment of MSCs in the infarcted myocardial area (Fig. 5). In the antibody staining of BrdU (Fig. 5A and B), angiogenesis and troponin I were found, and similarly, lymphocytic infiltration was present in the infarcted myocardium (Fig. 5C).

\section{Discussion}

Using the dog AMI model in the present study, intracoronary injection of bone marrow-derived MSCs into injured myocardium significantly improved cardiac functions, including reduction of the infarcted area and increase of left ventricle (LVEF). Notably, DISA and gated SPECT can be used for assessment of viability of newly-formed myocardium following MSCs transplantation, and this is of importance in practice, as SPECT is significantly less expensive with longer-lived more easily-obtained radioisotopes, that that of PET $(11,13)$.

Previously, stem cell-based treatments of AMI have been a focus of investigation. Autologous bone marrow-derived MSCs have been widely utilized as a result of plasticity, availability and lack of immunological rejection or ethical issues $(14,15)$. However, poor survival of transplanted MSCs in the injured myocardial area is one barrier to the success of stem cell therapy for AMI (16). Previous studies have shown that improvement of the periinfarct milieu with pharmacological treatment, such as simvastatin and atorvastatin, is beneficial for the survival of implanted stem cells (17-19). In the present study, pharmacological treatment was not used for improvement of the microenvironment for implanted stem cells, but a suitable injection time was selected; MSCs were injected after $2 \mathrm{~h}$ of myocardial reperfusion with $90 \mathrm{~min}$ of LAD ligation. Immediate injection of MSCs may be better that that of implantation within a week after AMI.

DISA SPECT offers the advantage of obtaining information on myocardial perfusion using ${ }^{99 \mathrm{~m} T c-M I B I}$ and metabolism
Table II. Infarcted segment number change at different time points after mesenchymal stem cell implantation.

\begin{tabular}{lccc}
\hline Group & 1 week & 10 weeks & Change \\
\hline Control & 20 & 18 & 2 \\
Graft & 25 & 15 & 10 \\
\hline
\end{tabular}

Table III. Change of LVEF (\%) following mesenchymal stem cell implantation.

\begin{tabular}{lccc}
\hline Group & 1 week & 10 weeks & Diffference \\
\hline Control & $50.50 \pm 8.02$ & $56.50 \pm 7.24$ & $5.50 \pm 2.69$ \\
Graft & $53.80 \pm 9.58$ & $70.00 \pm 7.52^{\mathrm{a}}$ & $16.20 \pm 2.93^{\mathrm{a}}$ \\
\hline
\end{tabular}

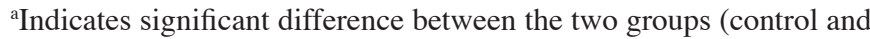
graft). LVEF, left ventricle.

using ${ }^{18} \mathrm{~F}-\mathrm{FDG}$ in a single study, with shortened duration of the procedure and an identical geometric registration of different isotope images (9). Direct comparison of the perfusion tracer ${ }^{99} \mathrm{~m}$ Tc-MIBI with metabolic tracer ${ }^{18} \mathrm{~F}-\mathrm{FDG}$ can determine the scarce and viable myocardium [Slarts et al (10)]. Comparing with PET perfusion tracers, such as ${ }^{15} \mathrm{O}$-water, ${ }^{13} \mathrm{~N}$-ammonia (which requires on-site cyclotron for radionuclide production) and ${ }^{82} \mathrm{Rb}$-rubidium (requires on-site generation for radionuclide production), DISA SPECT with ${ }^{99 \mathrm{~m} T c-M I B I} /{ }^{18} \mathrm{~F}-\mathrm{FDG}$ is much more widely used in hospital routine practice. Therefore, in the future, with rapid development of stem cell implantation-based therapy for treatment of AMI, DISP SPECT and gated SPECT will play more roles in assessment of cardiomyogenesis, angiogenesis, cardiac function and viable myocardial change following stem cell implantation for the injured heart.

In conclusion, direct intracoronary injection of bone marrow MSCs into injured myocardium in the experimental dog AMI model can significantly improve cardiac function with new vessel formation and myocyte-specific biomarker expression. In particular, the study further shows that DISA SPECT can be used for assessment of stem cells transplantation in the heart.

\section{Acknowledgements}

The present study was funded by a grant from the Fujian Provincial Youth Personnel of Scientific and Technology 
innovation item (no. 2005J070) and from the Affiliated Hospital of Inner Mongolia Medical College (no. nmgfy 200802). The authors thank Professors Xiejie Liu, Rongfang Shi and Zuoxiang He at Beijing Fuwai Hospital.

\section{References}

1. Keeley EC and Hillis LD: Primary PCI for myocardial infarction with ST-segment elevation. N Engl J Med 356: 47-54, 2007.

2. Martin-Rendon E, Brunskill SJ, Hyde CJ, Stanworth SJ, Mathur A and Watt SM: Autologous bone marrow stem cells to treat acute myocardial infarction: A systematic review. Eur Heart J 29: 1807-1818, 2008.

3. Penn MS (ed): Stem Cells and Myocardial Regeneration. 1st Edition. Humana Press, Totowa, p277, 2007.

4. Silva GV, Litovsky S, Assad JA, Sousa AL, Martin BJ, Vela D, Coulter SC, Lin J, Ober J, Vaughn WK, et al: Mesenchymal stem cells differentiate into an endothelial phenotype, enhance vascular density, and improve heart function in a canine chronic ischemia model. Circulation 111: 150-156, 2005.

5. Rodrigo SF, van Ramshorst J,Hoogslag GE, Boden H, Velders MA, Cannegieter SC, Roelofs H, Al Younis I, Dibbets-Schneider P, Fibbe WE, et al: Intramyocardial injection of autologous bone marrow-derived ex vivo expanded mesenchymal stem cells in acute myocardial infarction patients is feasible and safe up to 5 years of follow-up. J Cardiovasc Transl Res 6: 816-825, 2013.

6. Fukuda K and Fujita J: Mesenchymal, but not hematopoietic, stem cells can be mobilized and differentiate into cardiomyocytes after myocardial infarction in mice. Kidney Int 68: 1940-1943, 2005.

7. Castellani M, Colombo A, Giordano R, Pusineri E, Canzi C, Longari V, Piccaluga E, Palatresi S, Dellavedova L, Soligo D, et al: The role of PET with ${ }^{13} \mathrm{~N}$-ammonia and ${ }^{18} \mathrm{~F}$-FDG in the assessment of myocardial perfusion and metabolism in patients with recent AMI and intracoronary stem cell injection. J Nucl Med 51: 1908-1916, 2010.

8. Saraste A, Kajander S, Han C, Nesterov SV and Knuuti J: PET: Is myocardial flow quantification a clinical reality? J Nucl Cardiol 19: 1044-1059, 2012.

9. Rahmim A and Zaidi H: PET versus SPECT: Strengths, limitations and challenges. Nucl Med Commun 29: 193-207, 2008.
10. Slart RH, Bax JJ, van Veldhuisen DJ, van der Wall EE, Dierckx RA and Jager PL: Imaging techniques in nuclear cardiology for the assessment of myocardial viability. Int J Cardiovasc Imaging 22: 63-80, 2006.

11. Slart RH, Bax JJ, van Veldhuisen DJ, van der Wall EE, Irwan R, Sluiter WJ, Dierckx RA, de Boer J and Jager PL: Prediction of functional recovery after revascularization in patients with chronic ischaemic left ventricular dysfunction: Head-to-head comparison between ${ }^{99 \mathrm{~m}} \mathrm{Tc}$-sestamibi/ ${ }^{18} \mathrm{~F}$-FDG DISA SPECT and ${ }^{13} \mathrm{~N}$-ammonia/ $/{ }^{18} \mathrm{~F}-\mathrm{FDG}$ PET. Eur J Nucl Med Mol Imaging 33: 716-723, 2006

12. De Boer J, Slart RH, Blanksma PK, Willemsen AT, Jager PL, Paans AM, Vaalburg W and Piers DA: Comparison of ${ }^{99 \mathrm{~m}} \mathrm{Tc}$-sestamibi- ${ }^{18} \mathrm{~F}$-fluorodeoxyglucose dual isotope simultaneous acquisition and rest-stress ${ }^{99 \mathrm{~m}} \mathrm{Tc}$-sestamibi single photon emission computed tomography for the assessment of myocardial viability. Nucl Med Commun 24: 251-257, 2003.

13. Hutton BF: The origins of SPECT and SPECT/CT. Eur J Nucl Med Mol Imaging 41 (Suppl 1): S3-S16, 2014.

14. Psaltis PJ, Zannettino AC, Worthley SG and Gronthos S: Concise review: Mesenchymal stromal cells: Potential for cardiovascular repair. Stem Cells 26: 2201-2210, 2008.

15. Pittenger MF and Martin BJ: Mesenchymal stem cells and their potential as cardiac therapeutics. Circ Res 95: 9-20, 2004.

16. Robey TE, Saiget MK, Reinecke H and Murry CE: Systems approaches to preventing transplanted cell death in cardiac repair. J Mol Cell Cardiol 45: 567-581, 2008.

17. Song L, Yang YJ, Dong QT, Qian HY, Gao RL, Qiao SB, Shen R, He ZX, Lu MJ, Zhao SH, et al: Atorvastatin enhance efficacy of mesenchymal stem cells treatment for swine myocardial infarction via activation of nitric oxide synthase. PLoS One 8: e65702, 2013.

18. Yang YJ, Qian HY, Huang J, Li JJ, Gao RL, Dou KF, Yang GS, Willerson JT and Geng YJ: Combined therapy with simvastatin and bone marrow-derived mesenchymal stem cells increases benefits in infarcted swine hearts. Arterioscler Thromb Vasc Biol 29: 2076-2082, 2009.

19. Soboleva EL, Gabbasov ZA, Agapov AA, Akchurin RS, Saburova OS, Romanov YA and Smirnov VN: Circulating bone marrow stem/progenitor cells in vascular atherogenesis and in the noninvasive diagnosis of coronary stenosis. Exp Clin Cardiol 10: 184-188, 2005. 